\title{
SIMPRE1.2: considering the hyperfine and quadrupolar couplings and the nuclear spin bath decoherence
}

\author{
Salvador Cardona-Serra, ${ }^{2}$ Luis Escalera-Moreno, ${ }^{1}$ José J. Baldoví, ${ }^{1,3}$ Alejandro Gaita-Ariño, ${ }^{1}$ Juan M. \\ Clemente-Juan, ${ }^{1}$ Eugenio Coronado, ${ }^{1}$
}

Correspondence to: Alejandro Gaita-Ariño (E-mail: alejandro.gaita@uv.es)

\author{
${ }^{1}$ Instituto de Ciencia Molecular (ICMol) Universidad de Valencia, C/Catedrático José Beltrán 2, Paterna, 46980, \\ Spain \\ ${ }^{2}$ Trinity College Dublin, College Green, Dublin 2, Ireland \\ ${ }^{3}$ Institut de Chimie Moléculaire et des Matériaux d'Orsay, CNRS, Université de Paris Sud 11, 91405 Orsay Cedex, \\ France
}

\begin{abstract}
SIMPRE is a fortran77 code which uses an effective electrostatic model of point charges to predict the magnetic behavior of rare-earth-based mononuclear complexes. In this manuscript, we present SIMPRE1.2, which now takes into account two further phenomena. Firstly, SIMPRE now considers the hyperfine and quadrupolar interactions within the rare-earth ion, resulting in a more complete and realistic set of energy levels and wave functions. Secondly, and in order to widen SIMPRE's predictive capabilities regarding potential molecular spin qubits, it now includes a routine that calculates an upper-bound estimate of the decoherence time considering only the dipolar coupling between the electron spin and the surrounding nuclear spin bath. Additionally, SIMPRE now allows the user to introduce the crystal field parameters manually. Thus, we are able to demonstrate the new features using as examples (i) a Gd-based mononuclear complex known for its properties both as a Single lon Magnet and as a coherent qubit and (ii) an Er-based mononuclear complex.
\end{abstract}

\section{Introduction}

Dealing with the quantum nature of the spin constitutes both the challenge and the strength of molecular magnetism. This is why it has produced tools and materials that have served to enrich several neighbouring fields that also consider the spin as a quantum object, from molecular spintronics to quantum computing. ${ }^{1,2}$ Quantum effects have been harnessed with outstanding success using mononuclear Single Molecule Magnets (SMMs), also known as Single Ion Magnets (SIMs). For example, the read-out and manipulation of a single nuclear spin using a molecular transistor have been demonstrated using a lanthanoid
SIM. ${ }^{3,4}$ It even has been possible to perform a single-molecule experiment to quantify the magnetic interaction between a single radical spin and an individual SMM. ${ }^{5}$ The most recent developments in terms of molecular spin qubits have been the preparation of magnetic molecules with longer decoherence times by avoiding interaction with both phonons and neighbouring spins, using different strategies. ${ }^{6,7}$ It is expected that that improved theoretical modelling will serve to guide further advances in this field.

The theoretical modelling of the magnetic properties of SIMs deals fundamentally with the ligand field Hamiltonian, as this is the interaction that governs the energy level 
splitting from $2 \mathrm{~K}$ up to (and above) room temperature, always within the ground spinorbit multiplet. The two main approaches are (i) CAS(SCF/PT2) + RASSI-SO ab initio calculations or (ii) effective electrostatic models, the latter mainly with the SIMPRE package ${ }^{8}$ and the REC model. ${ }^{9,10}$ Both the $a b$ initio and the electrostatic approach are in principle capable of estimating the ligand field, although both are known to suffer from serious limitations even for this task. ${ }^{11-13}$ Some of these limitations in the theoretical modelling of lanthanoid complexes are fundamental, while others can be corrected, and this is the purpose of the present work.

One of these non-fundamental limitations is neglecting all nuclear spin terms. It is true that hyperfine coupling between the electronic and nuclear spin of the lanthanoid ion operates at temperatures around or below $1 \mathrm{~K}$, but this is precisely the temperature range where most interesting experiments are performed both in terms of single ion magnetism and in terms of coherent quantum manipulation. Indeed, nuclear spin levels were known to be crucial for the behavior of rare earth ions since the solidstate experiments that inspired and predated their current potential as $\mathrm{SMMs}^{14}$ and as molecular spin qubits, ${ }^{15}$ respectively. The extension of SIMPRE presented herein considers both the nuclear quadrupole term and the hyperfine coupling explicitly, solving this issue.

Hyperfine coupling is most intense with the nuclear spin of the lanthanoid ion, but the collective effect of the coupling with the nuclear spin bath is one of the three main sources of decoherence in magnetic molecules. In fact, the nuclear spin bath governs quantum decoherence at low enough temperatures and high enough dilution, ${ }^{16}$ the conditions that are routinely seeked to maximize the coherence time. $^{6,17}$ It is straightforward to estimate this contribution to decoherence given the expectation values of the magnetic moments in the two qubit states, which in turn can be derived from the wave functions. Thus, the current extension of SIMPRE also estimates decoherence caused by the nuclear spin bath. This extension has already been used in two published works: a study of the design of coherent molecular spin qubits based on (near) cubic complexes ${ }^{18}$ and a proposal on the use of polypeptides for the organization of SIMs and/or spin qubits. ${ }^{19}$ For clarity, in this work we will use two new examples to illustrate the use of SIMPRE1.2: a Gd-based mononuclear complex known for its properties both as a Single lon Magnet and as a coherent qubit and an Er-based mononuclear complex.

\section{Theoretical background}

We shall start by briefly revising the theoretical model that constitutes the foundations of SIMPRE. ${ }^{8(b)}$ Building upon that basis, we will detail the strategy of SIMPRE1.2 to deal with the hyperfine and quadrupolar couplings between the electron and nuclear spins of the lanthanoid ion and with the decoherence time due to the surrounding nuclear spin bath.

SIMPRE solves a Crystal Field (CF) Hamiltonian where the CF parameters for the ground J-multiplet are obtained by an electrostatic model that describes the ligands as point charges around the central ion placed at the origin of the system of coordinates. Such Hamiltonian expressed in terms of the Extended Stevens Operators $(E S O s)^{20}$ takes the general form:

$\hat{H}_{c f}=\sum_{k=2,4,6} \sum_{q=-k}^{k} B_{k}^{q} \hat{O}_{k}^{q}=\sum_{k=2,4,6} \sum_{q=-k}^{k} a_{k}\left(1-\sigma_{k}\right) A_{k}^{q}\left\langle r^{k}\right\rangle \hat{O}_{k}^{q}$

(1)

where $k$ (for $f$ - elements, $k=2,4,6$ ) is the order (also called rank or degree) and $q$ is the operator range, that varies between $k$ and $-k$, of the Stevens operator equivalents as defined by Ryabov in terms of the angular momentum operators $J_{ \pm}$and $J_{z}{ }^{21}$ (where the components $O_{k}^{q}(c)$ and $O_{k}^{q}(s)$ correspond to 
the ESOs with $q \geq 0$ and $q<0$, respectively). ${ }^{21}$ $B_{k}^{q}\left(A_{k}^{q}\right)$ are the crystal field parameters. $a_{k}$ are the tabulated $\alpha, \beta$ and $\gamma$ Stevens coefficients ${ }^{22}$ for $k=2,4,6$, respectively, which are tabulated and depend on the number of $f$ electrons, $\sigma_{k}$ are the Sternheimer shielding parameters ${ }^{23}$ of the $4 f$ electronic shell and $\left\langle r^{k}\right\rangle$ are the expectation values of the radius. ${ }^{23}$

The $A_{k}^{q}$ crystal field parameters are determined by the following relations:

$$
\begin{gathered}
A_{k}^{0}=\frac{4 \pi}{2 k+1} \sum_{i=1}^{N} \frac{z_{i} e^{2} Z_{k 0}\left(\theta_{i}, \varphi_{i}\right)}{R_{i}^{k+1}} p_{k 0} \\
A_{k}^{q}=\frac{4 \pi}{2 k+1} \sum_{i=1}^{N} \frac{z_{i} e^{2} Z_{k q}^{c}\left(\theta_{i}, \varphi_{i}\right)}{R_{i}^{k+1}} p_{k q} \\
\text { for } q>0 \\
A_{k}^{q}=\frac{4 \pi}{2 k+1} \sum_{i=1}^{N} \frac{z_{i} e^{2} Z_{k|q|}^{s}\left(\theta_{i}, \varphi_{i}\right)}{R_{i}^{k+1}} p_{k|q|} \\
\text { for } q<0
\end{gathered}
$$

where $R_{i}, \theta_{i}$ and $\varphi_{i}$ are the effective polar coordinates of the point charges, and $z_{i}$ is the effective point charge, associated to the $i^{\text {th }}$ donor atom with the lanthanoid at the origin. $N$ is the number of ligands; $e$ is the electron charge, $Z_{k q}$ are the tesseral harmonics expressed in terms of the spherical coordinates for the $i^{\text {th }}$ donor atom and $p_{k q}$ are the prefactors of the spherical harmonics.

From the relations ${ }^{24}$ between the ESOs and Wybourne operators, the Stevens CFPs and the Wybourne CFPs are obtained via the following conversion relations:

$$
\begin{gathered}
\lambda_{k 0} A_{k}^{0}\left\langle r^{k}\right\rangle=B_{k}^{0} \\
\lambda_{k q} A_{k}^{q}\left\langle r^{k}\right\rangle=\operatorname{Re} B_{k}^{q} \text { for } q>0 \\
\lambda_{k|q|} A_{k}^{q}\left\langle r^{k}\right\rangle=\operatorname{Im} B_{k}^{|q|} \text { for } q<0
\end{gathered}
$$

Here a bug in the output has been corrected: the signs of the Stevens's Bkq parameters were wrong in some instances in the output of SIMPRE1.1. This bug, which did not affect the prediction of actual physical properties in the output, is now corrected.

To calculate the magnetic properties, SIMPRE introduces the interaction between the electron spin and an external magnetic field along the z-direction via a Zeeman term $\hat{H}_{Z E}$ :

$$
\hat{H}_{Z E}=g_{J} \mu_{B} B_{z} \cdot \hat{J}_{z}
$$

where $g_{J}$ is the Landé g-factor for the ground $J$ - multiplet, $\mu_{B}$ is the Bohr magneton, $B_{z}$ is the external magnetic field along the z-direction and $\hat{J}_{z}$ is the $z$-component of the total electronic angular momentum operator. By manually changing the orientation of the complex, the user can effectively change the direction of the magnetic field.

SIMPRE1.2 includes both the hyperfine interaction between the electron spin of the lanthanoid ion and its own nuclear spin and the quadrupolar interaction to produce a more detailed description of the energy level spectrum at very low (sub-Kelvin) energies. This interaction is accounted for by the Hamiltonian:

$$
\hat{H}=A_{\|} \hat{J}_{z} \cdot \hat{I}_{z}+A_{\perp}\left(\hat{J}_{x} \cdot \hat{I}_{x}+\hat{J}_{y} \cdot \hat{I}_{y}\right)+P \hat{I}_{z}^{2}
$$

where $A_{\|}$and $A_{\perp}$ are the parallel and perpendicular hyperfine parameters, $P$ is the nuclear electric quadrupole parameter, $\hat{J}_{x}, \hat{J}_{y}$ and $\hat{J}_{z}$ act on the electron spin, and $\hat{I}_{x}, \hat{I}_{y}$ and $\hat{I}_{z}$ act on the nuclear spin.

As they will be needed to estimate the decoherence created by the nuclear spin bath, the code also calculates the expectation values $\left\langle\hat{J}_{\alpha}\right\rangle(\alpha=x, y, z)$ of any two qubit states $\{|0\rangle,|1\rangle\}$ specified by the user. These are calculated from the two wave functions $\Psi$ that 
represent those two qubit states by means of the Pauli matrices $\sigma_{\alpha}$ :

$$
\left\langle\hat{J}_{\alpha}\right\rangle=\left\langle\Psi\left|\sigma_{\alpha}\right| \Psi\right\rangle
$$

Taking as an input the coordinates of the nuclei in the vicinity of the lanthanoid complex together with those expectation values, SIMPRE1.2 calculates the dipolar magnetic field $\vec{B}(\vec{r}, \vec{m})$ felt by each nucleus:

$$
\vec{B}(\vec{r}, \vec{m})=\frac{\mu_{0}}{4 \pi}\left(3 \frac{(\vec{m} \cdot \vec{r}) \vec{r}}{|\vec{r}|^{5}}-\frac{\vec{m}}{|\vec{r}|^{3}}\right)
$$

where $\vec{r}$ is the vector connecting the nucleus of the bath and the lanthanoid ion, $\vec{m}=-\mu_{B} g_{J} \vec{J}$ is its electronic magnetic moment and $\mu_{0}, \mu_{B}$ are the magnetic constant and the Bohr magneton, respectively. The magnitude of the magnetic moment $\vec{m}$ is calculated as a function of $\left\langle\hat{J}_{\alpha}\right\rangle(\alpha=x, y, z)$ which, in general, will be different for the two qubit states $\{|0\rangle,|1\rangle\}$. The magnetic field $\vec{B}(\vec{r}, \vec{m})$ felt by the nucleus $k$ at coordinates $\vec{r}$ results in a dipolar hyperfine interaction energy $E$ :

$$
\begin{gathered}
E(k, \vec{r}, \vec{m})=\gamma_{N}(k) B(\vec{r}, \vec{m}) \\
\gamma_{N}(k)=\mu_{N} g_{N}(k)
\end{gathered}
$$

where $\mu_{N}$ is the nuclear magneton and $g_{N}(k), \gamma_{N}(k)$ are the Landé g-factor and the gyromagnetic ratio of the nucleus $k$, respectively.

When the qubit is in some given state $\alpha|0\rangle+\beta|1\rangle$, where $|\alpha|^{2}+|\beta|^{2}=1$, a finite nuclear spin bath with $\mathrm{N}$ nuclei gives rise to a set of $\prod_{k=1}^{N}\left(2 I_{k}+1\right)$ states, where $I_{k}$ is the spin of the $k^{\text {th }}$ nucleus. The energy associated with each one of these states can be expressed in terms of (8). The model ${ }^{16(b)}$ employed to estimate the nuclear decoherence time assumes that the density of states as a function of energy has a Gaussian line shape, since as $N$ becomes larger and larger, by the Central Limit Theorem, it converges to a Gaussian distribution. Then, the half-width $E_{0}$ of such a Gaussian distribution can be calculated as:

$$
E_{0}^{2}=\sum_{k} \frac{I_{k}+1}{3 I_{k}}\left(\omega_{k} I_{k}\right)^{2}
$$

where $\omega_{k}=E_{0}^{k}-E_{1}^{k}$, being $E_{0}^{k}$ and $E_{1}^{k}$ the energies (8) produced by the two qubit states $\{|0\rangle,|1\rangle\}$ for the $k^{\text {th }}$ nucleus. It is important to take this into consideration: if the density of states resulting from the nuclear spin bath cannot be approximated by a Gaussian distribution, the present method is not adequate.

Assuming a high - field regime, which means that $\Delta_{0}>>E_{0}$, where $\Delta_{0}$ is the energetic splitting in between the two qubit states, this dynamics can be solved perturbatively and, thus, the decoherence time $\tau$ due to the nuclear spin bath is estimated as:

$$
\tau=2 \hbar \frac{\Delta_{0}}{\sum_{k} \frac{I_{k}+1}{3 I_{k}}\left(\omega_{k} I_{k}\right)^{2}}
$$

In practice, the high - field condition is generally satisfied at rather low fields for EPR standards $(0.1 \mathrm{~T})$, but it does mean that a theoretical decoherence time at zero - or ultralow - fields cannot be estimated by these procedure.

\section{Organization of the Code and Data Flow}

Let us now explain how the new SIMPRE code is organized as well as what new 
subroutines and options have been incorporated.

Figure 1 depicts the scheme of the data flow of SIMPRE1.2. The boxes with continuous lines are the ones already present in SIMPRE1. $1^{8}$, while the ones with dashed lines correspond to the new subroutines and inputs incorporated to SIMPRE1.2. The program is started by including the input files simpre.par and simpre.dat. simpre.par cointains switches to choose among certain options:

(i) a choice between different energy units (cm-1, meV or K) and coordinate systems (Cartesian or spherical),

(ii) whether or not the molecule is rotated to simplify the ground wave function,

(iii) whether or not the magnetic susceptibility and the magnetization should be calculated,

(iv) whether or not the crystal field parameters should be manually introduced and

(v) whether or not the user desires to estimate the decoherence time due to a nuclear spin bath.

It also includes as parameters the dimension of the Hamiltonian matrix and the maximum number of charges to be used to model the coordination sphere.

simpre.dat is the file where the user introduces the coordinates and magnitudes of the point charges and specifies the lanthanoid ion, the hyperfine and quadrupolar parameters, and the magnitude of the applied magnetic field in the $\mathrm{Z}$ direction.

If thus choses in simpre.par, the user can manually introduce the crystal field parameters in the new input file simpre.bkq. This can be useful in case these parameters have already been determined experimentally or if the user wants to make a theoretical exploration. Otherwise, the parameters will be calculated by SIMPRE from the point charges, as it was done in previous versions of the program.

Using this input, the subroutines BKQ and ENE, together with the subroutines HYPE, HYPA, QUAD and ZEE, solve the Hamiltonian problem. BKQ calculates (or reads from simpre.bkq) the crystal field parameters, ENE builds and diagonalizes the Hamiltonian matrix, HYPE takes into account the perpendicular hyperfine interaction between the electron and nuclear spins of the lanthanoid (for simplicity, both $X$ and $Y$ directions are considered equal), while HYPA considers the parallel one, QUAD accounts for the quadrupolar interaction, and ZEE includes the electron Zeeman Hamiltonian. These new three contributions incorporated in SIMPRE1.2 are then included in the Hamiltonian matrix to be diagonalized within ENE. If one of the ROTA options is switched on in simpre.par, the subroutine will rotate the molecule by using a pre-defined angular grid and will rediagonalize the Hamiltonian matrix at each step until finding the orientation that gives either

(a) the most compact expression for the ground state wave function,

(b) the most intense magnetization at the field specified in simpre.dat i.e. the easy axis of magnetization or

(c) the least intense magnetization at this same field i.e. the hard magnetization axis.

Finally, the subroutine ENE creates the output file simpre.out that contains

(i) the input information for verification,

(ii) the calculation results: energies, wave functions and crystal field parameters and

(iii) possible errors and warnings generated along the execution.

At this point, depending on the switches activated in simpre.par, the program will either stop or proceed to calculate magnetic susceptibility and/or magnetization. In the latter case, the subroutines SUS and MAG will be invoked, and these in turn will invoke the subroutine ZEE to include the electron Zeeman Hamiltonian. Their results are written in sus.out and mag.out, respectively. 


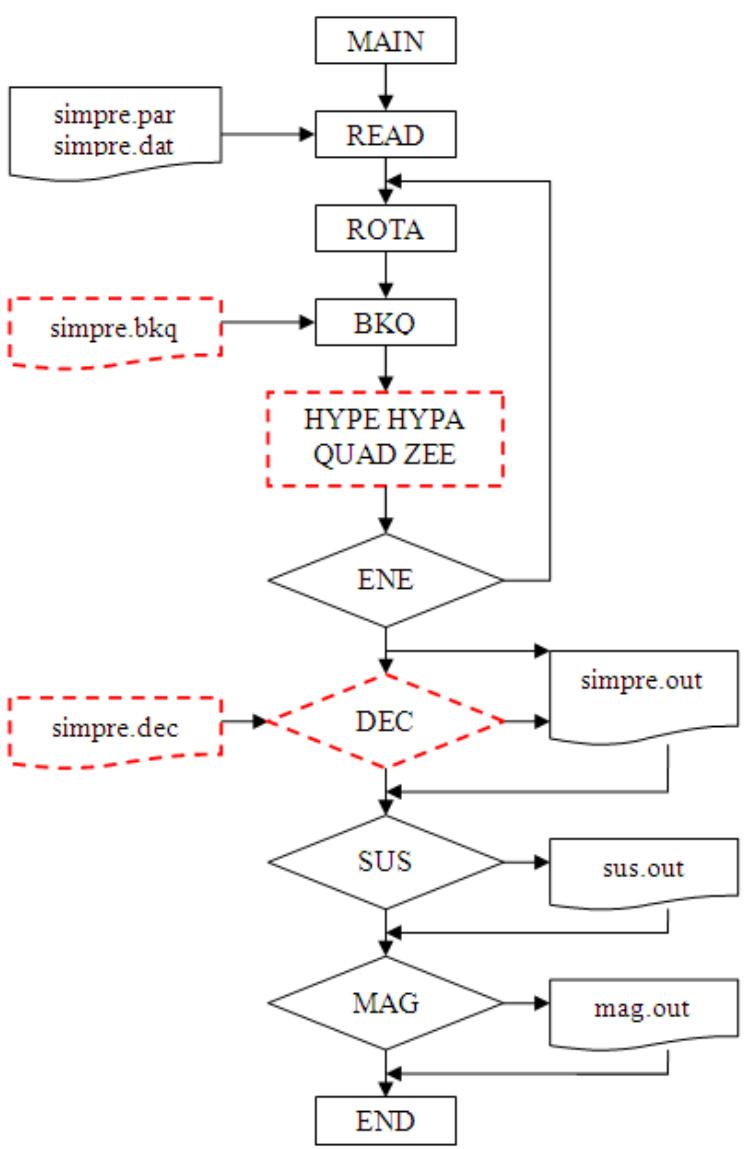

Figure 1. SIMPRE code organization showing the different subroutines and the data flow between them.

SIMPRE1.2 includes another new subroutine to estimate the decoherence time due to a nuclear spin bath. The subroutine DEC calculates such an estimate and the coordinates of the bath are written in the input file simpre.dec. This file is divided into as many blocks as different isotopes are present in the bath. Each block corresponds to a certain isotope and contains atomic coordinates together with the occupancy factor for each atom. SIMPRE1.2 considers the occupancy of every crystallographic position to proportionally estimate the contribution of each atom to the nuclear spin decoherence. The estimated decoherence time is found in simpre.out. The code of SIMPRE1.2 includes the gyromagnetic ratios of the most important isotopes for our purposes. To simulate the spin bath, SIMPRE1.2 distinguishes between protium and deuterium, as hydrogen is often the main contributor to the nuclear spin bath. The code explicitly includes the data corresponding to the isotopes that constitutes practically $100 \%$ of the natural abundance for N, F, Na and P. Nitrogen is often in the coordination sphere, while fluorine and sodium are common countercations. Phosphorus is included because of its importance in different polyoxometalates, and because it also displays a relatively large nuclear magnetic momentum. For $\mathrm{Cl}, \mathrm{K}$, and $\mathrm{W}$, the data assumes the natural distribution and thus it is a weighted average of the different isotopes. Chlorine and potassium, again, are common countercations, and tungsten, despite its low contribution, is relevant in polyoxometalates. Finally, the user can introduce a number of user-defined isotopes (see the online manual for more details).

As SIMPRE1.2 includes the lanthanoid nuclear spin $I$, the wave functions are now shown in the output file as linear combinations of the basis set elements $\left\{\left|m_{J}, m_{I}\right\rangle\right\}_{m_{J}, m_{I}}$ written in terms not only of the $m_{J}$ components but also in terms of the $m_{I}$ components.

\section{Application of the Program}

\section{Example 1: ${ }^{157} \mathrm{GdW}_{30}$}

The first example demonstrates how to estimate the decoherence time given a complex nuclear spin bath. The system employed to exemplify this new feature of SIMPRE1.2 is a Gd-based polyoxometalate $\left[\mathrm{GdW}_{30} \mathrm{P}_{5} \mathrm{O}_{110}\right]^{12-}$, abbreviated as $\mathrm{GdW}_{30}$, which has been reported to be both a potential coherent spin qubit ${ }^{25}$ and a Single Ion Magnet. ${ }^{26}$
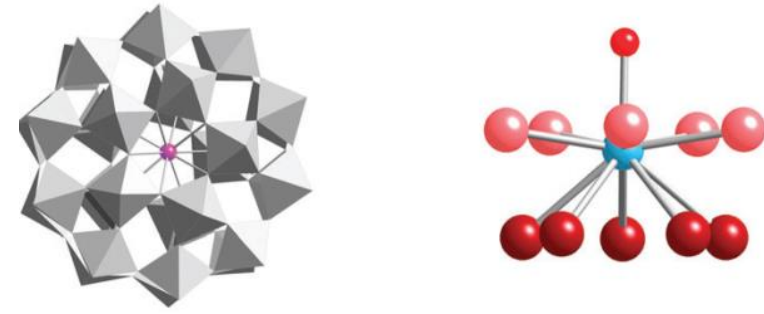
Figure 2. Views of $\mathrm{GdW}_{30}$ from above (left) and the first coordination sphere of $\mathrm{Gd}^{3+}$ from the side (right). The axial water is at $2.2 \AA$.

The first coordination sphere of $\mathrm{Gd}^{3+}$ is composed of ten $\mathrm{O}^{2-}$ anions of the proper polyoxometalate, together with an axial coordination water (see Figure 2). $\mathrm{Gd}^{3+}$ has no orbital angular momentum in the ground state, and thus the tabulated Stevens coefficients $\alpha$, $\beta, \gamma$ are zero for $\mathrm{Gd}^{3+}$, which means that the CF parameters calculated by the theoretical model of SIMPRE would be zero. Thus, for dealing with this ion one needs to introduce the values of the CF parameters by hand. In this case, the parameters were extracted from an experimental fitting ${ }^{26}$, and adapted to be used in the Hamiltonian (1) following the Wybourne notation. As a consequence, no point charges are required to be introduced in simpre.dat. Instead, a new user-defined lanthanoid is introduced with the electron and nuclear spins and $\mathrm{g}$ L Landé factor matching those of $\mathrm{Gd}^{3+}$, instead (see the manual for more details).

The CF parameters introduced in simpre.bkq were $B_{2}^{0}=0.0132 \mathrm{~cm}^{-1}$ and $B_{2}^{2}=0.0132 \mathrm{~cm}^{-1}$, the remaining ones are zero. In simpre.dat, we $\operatorname{set}^{27} A_{\|}=0.00053 \mathrm{~cm}$ 1, $A_{\perp}=0.00053 \mathrm{~cm}^{-1}, P=0.00000 \mathrm{~cm}^{-1}$ and a magnetic field of 0.32 T. In simpre.par, we left the default option of using the Sternheimer shielding parameters, and chose the two qubit states as the $1^{\text {st }}$ and the $9^{\text {th }}$ spin states, i.e. the first nuclear spin state of the first and second electronic spin states.

Coordinates and occupancy factors of the nuclear spin bath were taken from X-ray crystallographic data. As there is an effectively infinite number of nuclei in a crystal structure, a cutoff radius for the spin bath needs to be included in our calculations. As a criterion, we neglect every nucleus which, on average, is expected to produce less than $1 / 100^{\text {th }}$ of the effect produced by the two hydrogen nuclei of the water molecule directly coordinated to the gadolinium. As the dipolar energy interaction
(8) falls with the third power of the distance, this means that the cutoff radius is a factor of $100^{1 / 3}$ farther away than the nearest hydrogen nuclei. The bath is composed of hydrogen from the crystallization water molecules, $\mathrm{W}$ and $\mathrm{P}$ from neighbouring $\mathrm{GdW}_{30}$ complexes and potassium counter-cations.

We plot the estimated decoherence times by gradually considering the different elements, as this procedure allows distinguishing between the effects of the different nuclei (Figure 3). Tungsten nuclear spins produce a negligible amount of decoherence, although they are very close to the lanthanoid ion. Potassium countercations reduce decoherence time almost by an order of magnitude, and this effect of alkali metal counter-cations should always be taken into account. Phosphorus, which is inside the polyoxometalate, reduces the coherence time by almost two orders of magnitude. Finally, Hydrogen produces the most intense effect, although this can largely be mitigated by deuteration.

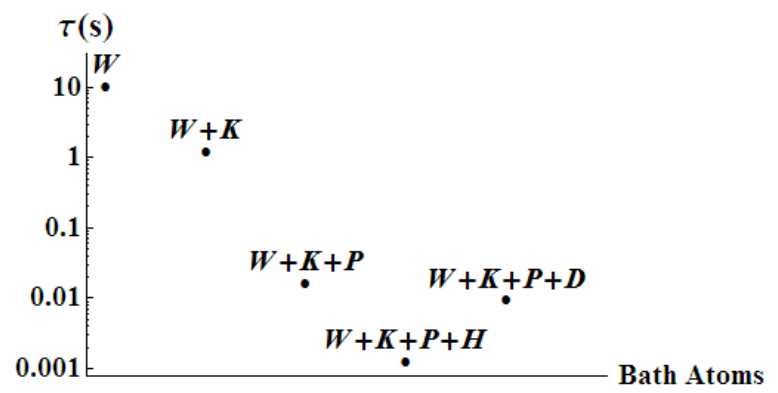

Figure 3. Evolution of the estimated decoherence time as different kinds of atoms are added to the nuclear spin bath.

Note that in these calculations the water molecule directly coordinated to the lanthanoid was not taken into account, as it cannot be properly described by our theoretical approach. As shown in Table 1, the estimated decoherence times are extremely dependent on whether or not deuterated water has been used and whether or not the closest water molecule has been considered. Moreover, table 2 shows that the corresponding dipolar interaction energies are also dominated by this water 
molecule. As a consequence, the Gaussian density of states of the rest of the spin bath is altered by the dipolar interaction with the water molecule directly coordinated to the lanthanoid (see Figures S1, S2). In practice, this means that the Gaussian approximation is no longer valid in this case and the decoherence caused by these two hydrogen should be characterized by different means.

Table 1. Estimated decoherence times due the nuclear spin bath surrounding the $\mathrm{GdW}_{30}$ complex.

\begin{tabular}{|c|c|c|}
\hline Dec. Time $(\mu \mathrm{s})$ & $\mathrm{H}_{2} \mathrm{O}$ & $\mathrm{D}_{2} \mathrm{O}$ \\
\hline $\mathrm{GdW}_{30}-$ water & 373.7 & 4565.1 \\
\hline $\mathrm{GdW}_{30}-$ no water & 1434.9 & 10562.7 \\
\hline
\end{tabular}

Table 2. Estimated dipolar interaction energy $E_{0}$ (see text) between the selected qubit and the nuclear spin bath surrounding the $\mathrm{GdW}_{30}$ complex.

\begin{tabular}{|c|c|c|}
\hline $\mathrm{E}_{0}(\mathrm{MHz})$ & $\mathrm{H}_{2} \mathrm{O}$ & $\mathrm{D}_{2} \mathrm{O}$ \\
\hline $\mathrm{GdW}_{30}-$ water & 2.83 & 0.80 \\
\hline $\mathrm{GdW}_{30}-$ no water & 1.43 & 0.53 \\
\hline
\end{tabular}

On the other hand, the calculated energetic gap $\Delta_{0}$ in between the two qubit states is 9.32 $\mathrm{GHz}$. As can be seen in Table 2, we observe that the high - field condition $\Delta_{0} \gg E_{0}$ is fulfilled.

The long decoherence times calculated here, in the order of magnitude of the millisecond, are comparable to those obtained in previous applications of the same software. ${ }^{28}$ They are, on the other hand, much longer than actual relaxation times determined experimentally. This it not surprising: the times calculated here should only be considered as an upper bound to the actual decoherence time at infinite dilution, that is, in absence of magnonbased decoherence. Comparing our results with the calculations for $\mathrm{Fe}_{8}$, depicted in Figure 2 in ref. 29 , one can observe that the decoherence caused by the nuclear spin bath, at moderate values of $\Delta_{0}$ (below $1 \mathrm{~K}$ ) is several orders of magnitude lower than decoherence caused by magnons. This means that, even at high dilutions, magnon decoherence can easily dominate over spin bath decoherence. This statement is valid for coordination complexes, but even more so for polyoxometalates, which are very poor in nuclear spins.

\section{Example 2: ${ }^{167}$ ErODA}

SIMPRE1.2 includes both the hyperfine and the quadrupolar interactions, giving rise to a better description of the energy level scheme. This example is intended to show this new feature by using a ${ }^{167} \mathrm{Er}^{3+}$ complex as a model system. This complex, with chemical formula $\left[\mathrm{Er}\left(\mathrm{C}_{4} \mathrm{H}_{4} \mathrm{O}_{5}\right)_{3}\right]^{3-}$ and abbreviated as ErODA, is shown in Figure 4.

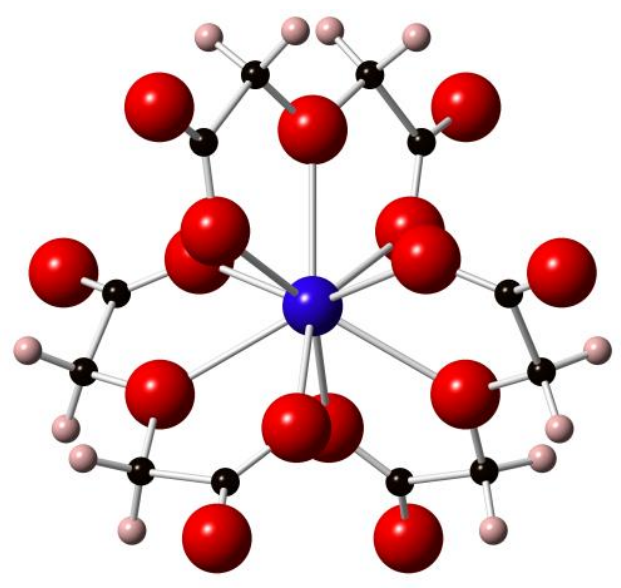

Figure 4. Ball-and-stick representation of the ErODA complex. Blue: Erbium, Red: Oxygen, Black: Carbon, Pale pink: Hydrogen.

In this example, SIMPRE will calculate the CF parameters from effective point charges at given coordinates. The nine oxygen atoms coordinating the ${ }^{167} \mathrm{Er}^{3+}$ ion will be described as effective point charges with a magnitude of $Z=$ 0.0851833 . Coordinates are shown in Table 3.

Table 3. Spherical coordinates $r, \theta, \varphi$ of the point charges employed in the description of the ErODA complex.

\begin{tabular}{l|l|l}
$r$ & $\theta$ & $\varphi$ \\
\hline
\end{tabular}




\begin{tabular}{|c|c|c|}
\hline 1.3376553 & 90.000 & 330.000 \\
\hline 1.3376553 & 90.000 & 210.000 \\
\hline 1.3376553 & 90.000 & 90.000 \\
\hline 1.2392742 & 46.2527317 & 276.7191128 \\
\hline 1.2392742 & 133.7472683 & 263.2808872 \\
\hline 1.2392742 & 133.7472683 & 23.2808871 \\
\hline 1.2392742 & 46.2527317 & 156.7191129 \\
\hline 1.2392742 & 46.2527317 & 36.7191129 \\
\hline 1.2392742 & 133.7472683 & 143.2808871 \\
\hline
\end{tabular}

In simpre.par we also accepted the default use of the Sternheimer shielding parameters; and in simpre.dat the hyperfine and quadrupolar parameters ${ }^{30}$ were set to be $A_{\|}=0.00520 \mathrm{~cm}^{-1}, A_{\perp}=0.03140 \mathrm{~cm}^{-1}$ and $P=0.00300 \quad \mathrm{~cm}^{-1}$. For demonstration purposes, these parameters were switched on successively.

Figure 5 depicts the energy level scheme of the lowest 16 spin states, accounting for the 8 nuclear spin states of the two lowest electron spin states. We show how the state distribution changes as the hyperfine and quadrupolar parameters are progressively turned on. In the first column, all three parameters are zero. In the second column, only $A_{\|}$is working, and a typical exchange-like scheme is found, with energy levels equi-spaced by the Landè interval. In the third column, both $A_{\|}$and $A_{\perp}$ are working, and this extradiagonal term results in (minor) tunneling splittings. Finally, in the fourth column all three parameters are working, something that maintains the order of the energy levels but significantly alters their spacing. For the full Hamiltonian, the $m_{J}$ and $m_{I}$ expectation values of the doublets are displayed in the Figure.

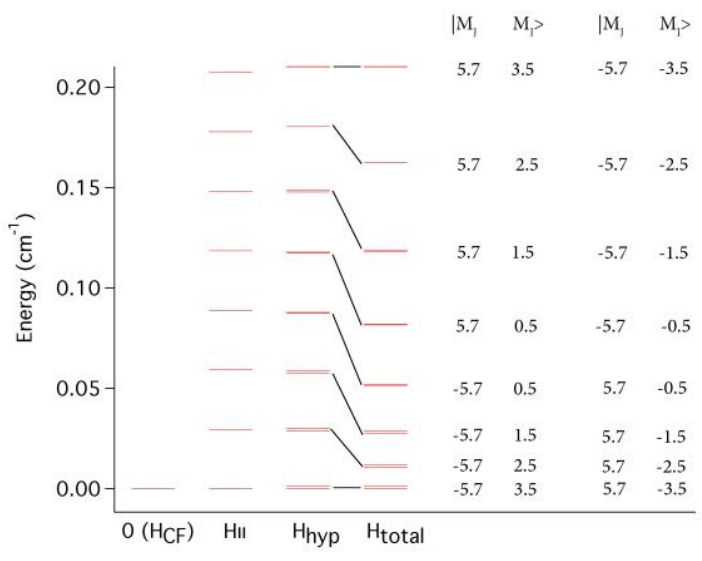

Figure 5. Energy level scheme of the lowest 16 spin states in the ErODA complex. The effect of the different terms of the Hamiltonian is shown by progressively switching on the parallel hyperfine coupling, the perpendicular hyperfine coupling and the quadrupolar terms. $m_{J}$ and $m_{I}$ expectation values correspond to the full Hamiltonian.

\section{Conclusions}

The SIMPRE package, employed to apply the REC model, has proven to be a useful tool to describe and rationalize magnetic properties of lanthanoid complexes, and even to guide the preparation of novel Single Ion Magnets. SIMPRE1.2 significantly extends these capabilities to the domain of molecular spin qubits. These improvements are mainly twofold. Firstly, SIMPRE1.2 considers the lanthanoid nuclear spin and therefore calculates a much more detailed energy level scheme, something crucial for the interpretation of EPR experiments. Secondly, SIMPRE1.2 also considers the coupling between the electron spin qubit to the nuclear spin bath and how this influences the relaxation time. As an additional improvement, the program now allows the automatic orientation of the molecule along either the easy or the hard axis of magnetization, and it also accepts CF parameters as an input. 


\section{Acknowledgments}

The present work has been funded by the EU (ERC Advanced Grant SPINMOL and ERC Consolidator Grant DECRESIM), the Spanish MINECO (grant MAT2014-56143-R, CTQ201452758-P), and the Generalitat Valenciana (Prometeo and ISIC Programmes of Excellence). A.G.-A. acknowledges funding by the MINECO (Ramón y Cajal contract). E.C. and J.J.B. acknowledge the Blaise Pascal International Chair for financial support. J.J.B. also thanks the Spanish MECD for an FPU predoctoral grant. L.E.M. acknowledges the Generalitat Valenciana for a VALi+D predoctoral grant.

Keywords: molecular magnetism, single ion magnets, point charges model, crystal field Hamiltonian, magnetic properties

((Additional Supporting Information may be found in the online version of this article.))

\section{References and Notes}

1. L. Bogani, W. Wernsdorfer, Nature Materials 2008, 7, $179-186$

2. (a) J. M. Clemente-Juan, E. Coronado, A. Gaita-Ariño, Chem. Soc. Rev. 2012, 41, 7464 - 7478 (b) G. Aromí, D. Aguilà, P. Gamez, F. Luis, O. Roubeau, Chem. Soc. Rev. 2012, 41, $537-546$

3. R. Vincent, S. Klyatskaya, M. Ruben, W. Wernsdorfer, F. Balestro, Nature 2012, $488,357-360$

4. S. Thiele, F. Balestro, R. Ballou, S. Klyatskaya, M. Ruben, W. Wernsdorfer, Science 2014, 344(6188), 1135 - 1138

5. M. Urdampilleta, S. Klyatskaya, M. Ruben, W. Wernsdorfer, ACS Nano 2015, 9(4), 4458 - 4464

6. K. Bader, D. Dengler, S. Lenz, B. Endeward, Shang-Da Jiang, $P$. Neugebauer, J. van Slageren, Nature Communications 2014, 5, 5304
7. M. Shiddiq, D. Komijani, Y. Duan, A. Gaita-Ariño, E. Coronado, S. Hill, submitted.

8. (a) J. J. Baldoví, S. Cardona-Serra, J. M. Clemente-Juan, E. Coronado, A. GaitaAriño, A. Palii, J. Comput. Chem. 2013, 34(22), 1961 - 1967 (b) J. J. Baldoví, J. M. Clemente-Juan, E. Coronado, A. Gaita-Ariño, A. Palii, J. Comput. Chem. 2014, 35(26), 1930 - 1934

9. J. J. Baldoví, J. J. Borrás-Almenar, J. M. Clemente-Juan, E. Coronado, A. GaitaAriño, Dalton Trans. 2012, 41, 13705 13710

10. J. J. Baldoví, A. Gaita-Ariño, E. Coronado, Dalton Trans. 2015, 44, $12535-12538$

11. J. J. Baldoví, Y. Duan, R. Morales, A. Gaita-Ariño, E. Ruiz, E. Coronado, 2015, submitted

12. K. Qian, J. J. Baldoví, Shang-Da Jiang, A. Gaita-Ariño, Yi-Quan Zhang, J. Overgaard, Bing-Wu Wang, E. Coronado, S. Gao, Chem. Sci. 2015, 6, $4587-4593$

13. J. J. Baldoví, J. M. Clemente-Juan, E. Coronado, A. Gaita-Ariño, Inorg. Chem. 2014, 53(20), 11323 - 11327

14. R. Giraud, W. Wernsdorfer, A. M. Tkachuk, D. Mailly, B. Barbara, Phys. Rev. Lett. 2001, 87(057203)

15. S. Bertaina, S. Gambarelli, A. Tkachuk, I. N. Kurkin, B. Malkin, A. Stepanov, B. Barbara, Nature Nanotechnology 2007, 2, $39-42$

16. (a) S. Takahashi, I. S. Tupitsyn, J. van Tol, C. C. Beedle, D. N. Hendrickson, P. C. E. Stamp, Nature 2011, 476, 76 - 79 (b) P. C. E. Stamp, I. S. Tupitsyn, Phys. Rev. B 2004, 69(014401)

17. C. J. Wedge, G. A. Timco, E. T. Spielberg, R. E. George, F. Tuna, S. Rigby, E. J. L. McInnes, R. E. P. Winpenny, S. J. Blundell, A. Ardavan, Phys. Rev. Lett. 2012, 108(107204)

18. J. J. Baldoví, L. E. Rosaleny, V. Ramachandran, J. Christian, N. S. Dalal, J. M. Clemente-Juan, Peng-Yang, U. 
Kortz, A. Gaita-Ariño, E. Coronado, Inorg. Chem. Front. 2015, 2, $893-897$

19. L. E. Rosaleny, A. Gaita-Ariño, Inorg. Chem. Front. 2015, DOI: 10.1039/C5QI00127G

20. (a) C. Rudowicz, C. Y. Chung, J. Phys. Condens. Matter 2004, 16, 5825 (b) C. Rudowicz, J. Phys. C. Solid State Phys. 1985, 18, 1415 (c) C. Rudowicz, J. Phys. C. Solid State Phys. 1985, 18, 3837 (erratum)

21. I. D. Ryabov, J. Magn. Reson. 1999, 140, 141

22. K. W. H. Stevens, Proc. Phys. Soc. 1952, 65, 209

23. S. Edvardsson, M. Klinterberg, J. Alloys Compd. 1998, 275, 233

24. C. Rudowicz, M. Karbowiak, Coord. Chem. Rev. 2015, 287, $28-63$

25. J. J. Baldoví, S. Cardona-Serra, J. M. Clemente-Juan, E. Coronado, A. GaitaAriño, H. Prima-García, Chem. Commun. 2013, 49, $8922-8924$
26. M. J. Martínez-Pérez, S. Cardona-Serra, C. Schlegel, F. Moro, P. J. Alonso, H. Prima-García, J. M. Clemente-Juan, M. Evangelisti, A. Gaita-Ariño, J. Sesé, J. van Slageren, E. Coronado, F. Luis, Phys. Rev. Lett. 2012, 108, 247213

27. J. M. Baker, G. M. Copland, B. M. Wanklyn, J. Phys. C (Solid St. Phys.) 1969, Ser. 2, Vol. 2, $862-869$

28. L. E. Rosaleny, A. Gaita-Ariño, Inorg. Chem. Front. 2015, DOI: 10.1039/C5QI00127G

29. S. Takahashi, I. S. Tupitsyn, J. van Tol, C. C. Beedle, D. N. Hendrickson, P. C. E. Stamp, Nature 2011, 476, $76-79$

30. G. S. Bogle, H. J. Duffus, H. E. D. Scovil, Proceedings of the Physical Society 1952, section A, volume 65, Number 9 , $760-761$ 


\section{GRAPHICAL ABSTRACT}

Salvador Cardona-Serra, Luis Escalera-Moreno, José J. Baldoví, Alejandro Gaita-Ariño, Juan M. ClementeJuan, Eugenio Coronado

SIMPRE1.2: considering the hyperfine and quadrupolar couplings and the nuclear spin bath decoherence

SIMPRE1.2 goes beyond Single Ion Magnet Magnetic Prediction. By considering the coupling between the electronic and the nuclear spins of the lanthanoid ion, it now provides a better description of the low-energy levels. By calculating dipolar interactions, it estimates the quantum decoherence created by the environmental nuclear spins in the crystal. In sum, it is now useful as a tool to provide a first inexpensive description of lanthanoid complexes as molecular spin qubits.

GRAPHICAL ABSTRACT FIGURE ((Please provide a square image to be produced at $50 \mathrm{~mm}$ wide by 50 $\mathrm{mm}$ high.))

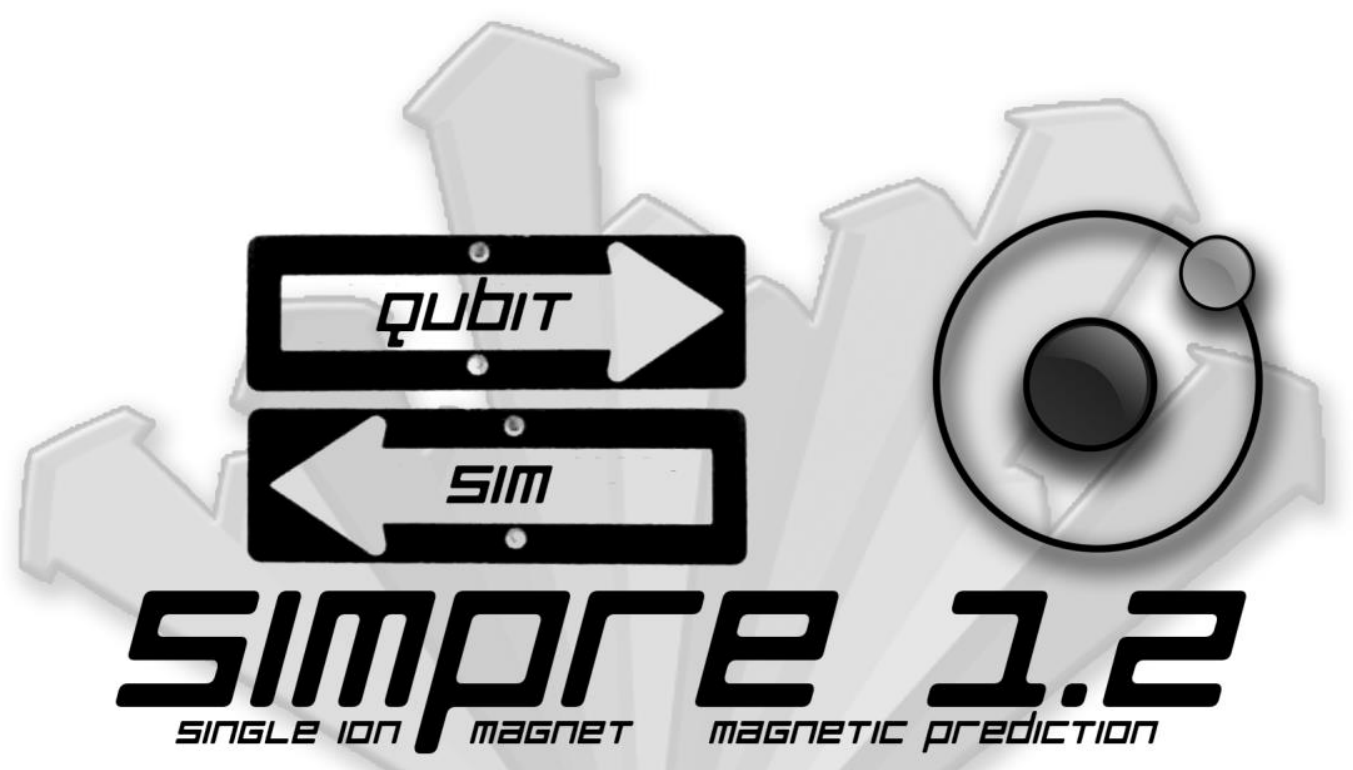

